\title{
Latife Yazigi: Abertura para o Mundo e para a Profissão
}

\author{
Regina Sonia Gattas Fernandes do Nascimento ${ }^{1}$ \\ ${ }^{1}$ Pontifícia Universidade Católica, SP, Brasil.
}

Resumo: Trata-se de um texto para homenagear a Profa, Dra. Latife Yazigi, professora e psicóloga de renomada importância para a área de Avaliação Psicológica. Possui uma notável carreira, com contribuição expressiva para o respeito pelos instrumentos de avaliação psicológica, para o reconhecimento da qualidade que os testes podem proporcionar ao trabalho na clínica e para profissionais de outras áreas da saúde, especialmente para médicos. Formada e doutorada pela USP, percorreu grandes distâncias para a formação complementar. Iniciou sua carreira no Brasil no Instituto de Psiquiatria da Faculdade de Medicina da USP e depois entrou para o Departamento de Psiquiatria e Psicologia Médica da Unifesp, no qual veio a se tornar Livre-docente e Professora Titular. Fez diversos cursos fora do país e fez um grande intercâmbio com grandes profissionais de outros países, convidando-os para ministrar cursos para psicólogos brasileiros. Coordenou cursos variados de graduação, especialização e pós-graduação, sempre com distinção e muita dedicação. Orientou inúmeras teses de mestrado e de doutorado, formando muitos profissionais na área da avaliação psicológica. No curso de especialização para psicólogos no Departamento de Psiquiatria e Psicologia Médica preparou diversos psicólogos para a atuação profissional, psicólogos estes que multiplicaram os conhecimentos recebidos e cujas carreiras testemunham sua formação. Proferiu muitas conferências. É autora de vários textos, tendo sido sua dedicação maior à produção de artigos científicos, nacionais e internacionais, especialmente na área da avaliação psicológica, com destaque ao método de Rorschach.

Palavras-chave: Latife Yazigi, Homenagem, Avaliação Psicológica. 


\title{
Latife Yazigi: an Opening to the World and to the Profession
}

\begin{abstract}
This text seeks to honor Latife Yazigi a Professor and Psychologist of renowned importance in psychological assessment. Dr. Latife has enjoyed an outstanding career with an expressive contribution to the fortification of the respect for the instruments of psychological evaluation. She recognized the quality the tests can provide to clinical work and to professionals from other areas of health, especially Medical Doctors. She walked great distances striving for complete qualification after receiving solid academic formation and a Doctorate from USP. She started her career in Brazil at the Institute of Psychiatry at the Medical University of USP, and then entered the Department of Medical Psychiatry and Psychology at UNIFESP where she became Full Tenured Professor and earned the title of Full Professor. She has participated in various courses outside of Brazil as well as administered an extensive exchange program with foreign professionals who were invited to come to Brazil to instruct psychologists of the country. She has coordinated postgraduate courses with distinction and devoted dedication while orientating numerous Masters and Doctorate theses, training many professionals in the area of psychological evaluation. In the graduate course for Psychologists at the Medical Psychiatry and Phycology Department, she prepared legions of professionals who have multiplied the knowledge they received and whose careers testify their training. She has lectured at a myriad of conferences. She is the author of numerous papers and national and international scientific articles on Psychological evaluation, especially the Rorschach test.
\end{abstract}

Keywords: Latife Yazigi, Homage, Psychological Assessment.

\section{Latife Yazigi: Apertura para el Mundo y para la Profesión}

Resumen: Se trata de un texto para homenajear a la Profesora, Dra. Latife Yazigi, profesora y psicóloga de renombrada importancia para el área de la Evaluación Psicológica. Posee una notable carrera, habiendo contribuido significativamente al respeto por los instrumentos de evaluación psicológica, al reconocimiento de la calidad que los tests pueden proporcionarle al trabajo en la clínica y a profesionales de otras áreas de la salud, particularmente a los médicos. Con título y doctorado de la USP, recorrió grandes distancias para complementar su formación. Inició su carrera en el Brasil, en el Instituto de Psiquiatría de la Facultad de Medicina de la USP y luego entró al Departamento de Psiquiatría y Psicología Médica de la Unifesp, en el cual se tornó Docente Libre y Profesora Titular. Hizo diversos cursos fuera del país y realizó un gran intercambio con importantes profesionales de otros países, invitándolos a dar cursos a psicólogos brasileños. Coordinó diversos cursos de graduación, especialización y posgrado, siempre con distinción y mucha dedicación. Orientó innumerables tesis de maestría e doctorado, formando muchos profesionales en el área de la evaluación psicológica. En el curso de especialización para psicólogos en el Departamento de Psiquiatría y Psicología Médica, preparó a diversos psicólogos, quienes multiplicaron los conocimientos recibidos y cuyas carreras son testimonio de su formación. Fue oradora en muchas conferencias. Es autora de varios textos, habiendo sido la producción de artículos científicos, nacional e internacionalmente, su dedicación más importante, especialmente en el área de la evaluación psicológica, especialmente el método de Rorschach.

Palabras clave: Latife Yazigi; Homenaje; Evaluación Psicológica; 
Sinto-me honrada e é um enorme prazer, mas considero também uma grande responsabilidade falar sobre a trajetória de vida acadêmica e pessoal da Profa. Dra. Latife Yazigi, que em muitos aspectos se entrelaçam e no nosso caso, nos entrelaçam. Uma honra por merecer estar próxima de uma pessoa com destaques nacional e internacional, com tantas contribuições para o engrandecimento e reconhecimento da área da Avaliação Psicológica, conferindo seriedade a esta área e revelando conhecimentos e resultados de pesquisas que incrementaram e incrementam o alcance dos conhecimentos. E o que dizer de sua ética em tudo o que faz e que tem marcado sua carreira? Considero que o que manifestarei neste documento representa a opinião de um grande número de colegas, que como eu, também estiveram próximas desta destacada professora, pesquisadora e psicóloga.

Uma responsabilidade por me propor a falar sobre uma trajetória tão complexa e com tantas produções e por ter que demonstrar de maneira fiel e clara o que ela já evidenciou com muito rigor e criatividade. Profa. Dra. Latife Yazigi tem como títulos acadêmicos: Doutora em Ciências pelo Instituto de Psicologia da Universidade de São Paulo (1973), Professora Titular e Livre-Docente do Departamento de Psiquiatria e Psicologia Médica da Escola Paulista de Medicina (Unifesp) (1978-2015), Visiting Scholar no Departament of Behavioral Sciences da University of Chicago (1981). (Yazigi, 1994)

Teve também diversos cargos de destaque no Brasil, dos quais podemos citar Membro do Comitê de Ciências Humanas da Fundação de Amparo à Pesquisa do Estado de São Paulo (2006-2013); fundadora e hoje participa do Conselho Consultivo da Associação Brasileira de Rorschach (ASBRo) desde 1993, Em 1998 e 1999 atuou como assessora técnica da Fundação de Amparo à Pesquisa do Rio Grande do Sul. Desde 1986 até o momento atual participa como Conselhos, Comissões e Consultoria, Assessoria da Coordenação de Aperfeiçoamento de Pessoal de Nível Superior (Capes).

Fora do Brasil, citamos sua atuação Entre 1997 até 2011, ocupou cargos de destaque na International Rorschach Society, tendo atuado como $1^{\text {a }}$ vice-presidente, $2^{\text {a }}$ vice-presidente por duas gestões e member at large; como Secretária Geral da Société Internationale de Psychopathologie Phénoméno-Structurale, na França entre 1993 e 1999 e vice-presidente entre 1999 e 2005. Foi também secretária geral do Partners of the Américas, no período de 1987 a 1988.
Logo no início de sua carreira esteve por um período na Argélia, onde atuou como psicossocióloga no Bureau National d'Études Économiques et Techniques, em 1971. Fez Pós-Doutorado em Psicologia no Department of Behavioral Sciences da University of Chicago, entre 1981 e 1983, tendo como orientador o Prof. Robert A. Buttler, Ph.D., e também frequentou um programa de Intercâmbio Internacional, no Laboratoire de Psychopathologie Cognitive Appliquée da Université de Liège, Bélgica, em 1982, com orientação de Meyer Timsit M.D.

Um prazer, porque este trabalho se mistura com sentimentos de afinidade e proximidade. Além da sincera amizade, Profa. Latife já abriu portas para mim e para muitos outros colegas no caminho da Psicologia, com grande generosidade. Nunca temeu ver aqueles que a acompanham e estão seu lado crescerem e também se sobressaírem. Muitos dos que hoje ocupam cargos de destaque na área da avaliação psicológica foram seus alunos ou orientandos.

Vamos começar falando da pessoa Latife Yazigi. Começo por mencionar sua grande capacidade para a compreensão da mente humana, seja como profissional, seja em seus relacionamentos pessoais. Como dito acima, destaca-se também sua generosidade com seus companheiros, amigos e familiares. Pessoa muito amorosa, vive rodeada por muitos. É muito cuidadosa com todos e sempre disposta a cooperar. Com sua inteligência e perspicácia sempre pronta a orientar. Seu bom senso está a dispor daqueles que precisam tomar decisões e a procuram para dividir suas dúvidas e angústias, aos que ela sempre deixa a liberdade e a autonomia para tomar suas decisões. Tem sido uma grande incentivadora das pessoas. Enfim, como pessoa, ou como orientadora apresenta tantas qualidades, que seria impossível esgotá-las, embora não possa deixar de mencionar... uma grande companheira de viagens.

Por outro lado, devemos destacar sua inteligência e criatividade. De maneira muito silenciosa, mas corajosa, conseguiu o respeito e reconhecimento de muitas autoridades do campo da Psicologia. Desbravadora de muitas áreas de atuação, modos de olhar para a Psicologia e em especial para a avaliação psicológica, construiu alianças com muitos profissionais - nacionais e internacionais - renomados. Vou exemplificar com um fato. Em 1981, quando eu me sentia ainda uma iniciante, fui para a minha primeira experiência em congresso internacional, em Washington, 
estimulada pela amiga Latife, que também era bastante jovem, mas já tinha inúmeros contatos com os pesquisadores de outros países. Seu nome já tinha um reconhecimento. Certo dia, durante este evento, Latife diz "amanhã vamos almoçar com Piotrowski" (um dos principais especialistas na história do método de Rorschach). E fomos. Que honra para ela, para mim e para algumas colegas brasileiras que também estavam. Destaco a presença de Lilian Picinelli nesta mesa. Esse foi o meu primeiro contato internacional, já com uma estrela de enorme grandeza. Latife, que vinha vencendo desafios e abrindo muitas portas, colocou o Brasil no caminho dos encontros internacionais, especialmente de avaliação psicológica, com acento significativo para o método de Rorschach. Seus horizontes são muito amplos, seu limite de vida pessoal e profissional é o mundo, o que mostra a abertura de sua posição frente à profissão e à vida.

Desde o início de sua carreira mostrou sua tendência a explorar novos ambientes, com sua incessante motivação para viajar para lazer, conhecimento e para engrandecer sua carreira, como logo em 1971 foi procurar experiência profissional na Argélia. Seus contatos internacionais são inúmeros. Com John Exner, desfrutou de reconhecimento, amizade e intercâmbios em diversas situações e trabalhos. Especialmente na época em que foi vice-presidente da International Rorschach Society, em que Exner era o presidente.

Para o Brasil trouxe Profa. Jerre Levy e Prof. Salvatore Maddi, ambos da Chicago University (1983); Prof. Mihaly Csikszentmihalyi, também da Chicago University (1984); K.W. Bash - na época presidente da International Rorschach Society (1985); Prof. Bryce Boyer - Psicanalista de São Francisco (1989); Meyer Timsit da Université de Liège (1990); David Ephraim, da Universidade Central da Venezuela (1991); Zena Helman e Jean Marie Barthelemy - da Universidade de Lile III (1993); Irving Weiner - da University of South Florida (2000); Anne Andronikof - da Université Paris Ouest Nanterre La Défense - UPX (2003); Montserrat Ros (Barcelona), Frida Roussel (Lausanne), Elizabeth Sorribas e Fernando Silberstein da Argentina. Em 2013, Piero Porcelli (Itália) e Tevfika Ikis (Turquia). Estes professores e ainda outros mais estiveram no Brasil ministrando cursos e contribuindo para ampliar a formação nas áreas das neurociências e avaliação psicológica, e trazendo benefícios a muitos especialistas destas especialidades bem como a evolução da Psicologia, cujos conhecimentos puderam alcançar níveis de atualização e possibilidade de acompanhar os conhecimentos de centros mais avançados.

Neste mesmo sentido destaca-se a organização do XII International Rorschach Congress, que em 1987 (Yazigi, \& Succar, 1987) ocorreu no Brasil, tendo a Profa. Latife como presidente. Foi a primeira e única vez que este congresso ocorreu no hemisfério sul. Foi um evento de expressiva importância para a comunidade Rorschach. Estiveram aqui presentes John Exner (Estados Unidos), Anne Andronikoff (França), Irving Weiner (Estados Unidos), Concépcion Sendin (Espanha), Nina Rausch de Traubenberg (França), Adriana Lis (Itália), Danilo Silva (Portugal), Elizabeth Teresa Sorribas e Fernando Silberstein (Argentina), Frieda Rossel (Suíça), Helena Lunazzi (Argentina), Howard Lerner (Estados Unidos), Julieta Lagomarsino (Uruguai), Meyer Timsit (Bélgica), Odile Husain (Canadá), Vera Campo (Espanha) e muitos outros. Foi uma oportunidade incrível para que os brasileiros pudessem estar próximos de tantas estrelas do Rorschach, que em grande número participaram deste evento, desde os grandes especialistas, até alunos de cursos de Psicologia ou de pós-graduação na área.

Sempre em busca de novos conhecimentos e aprimoramentos, Profa. Latife cruzou oceanos para completar sua formação. Além de sua primeira experiência na Argélia e do seu estágio pós-doutorado em Chicago, podemos destacar alguns dos cursos de extensão universitária que já frequentava no início de sua carreira. Diversos destes cursos foram na University of Chicago, nos Estados Unidos, dos quais mencionaremos Cerebral Asymmetry, em 1981 e em 1982, interesse que gerou sua pesquisa para o concurso para a Livre-Docência (Yazigi, 1994). Estes cursos pertenciam ao seu pós-doutorado. Seminars for Mental Health Professionals, na University of Illinois, nos Estados Unidos, em 1984. Mais recentemente, citamos o curso Assessing Managing And Treating Personality Disorder, no Specialized Training Service (STS), novamente nos Estados Unidos, em 2005; Research Training in Psychotherapy, na International Psychoanalytic Association (IPA), Inglaterra, também em 2005. Como se pode notar, seu interesse pela neuropsicologia, pela saúde mental e pela pesquisa estiveram sempre presentes em sua formação (Yazigi, 1994).

Tem sido, há muitos anos, assídua frequentadora dos Congressos Internacionais de Rorschach e também dos Summer Seminars da International Rorschach Society. Em ambas as reuniões é uma professora de bas- 
tante destaque e reconhecimento, para as quais tem trazido muitas contribuições com os resultados de suas pesquisas, mas já tendo também proferido conferências.

A carreira da Profa. Latife Yazigi no Brasil teve início em 1973, quando entrou como psicóloga no Instituto de Psiquiatria do Hospital das Clínicas da Faculdade de Medicina da Universidade de São Paulo (USP), local em que trabalhou até 1976 e onde desenvolveu pesquisas. Saiu desta Instituição como Diretora e Administradora do Serviço de Psicologia.

Em 1974 iniciou sua carreira de docente. Começou como professora da Pontifícia Universidade Católica (PUC) de Campinas. Nesta faculdade ensinou Rorschach e Psicodiagnóstico, em nível de graduação e pós-graduação. No mesmo ano ingressou como professora da Sociedade de Rorschach de São Paulo, onde permaneceu até 1981 e também exerceu os cargos de secretária, coordenadora e diretora.

Neste mesmo período, entre 1978 e 1981, fez parte do corpo docente pós-graduação do Instituto Metodista de Ensino Superior. Lá foi professora, orientadora e coordenadora do mestrado desta instituição de ensino. Foi também membro da Comissão de Pós-graduação. Ministrou aulas sobre Transtornos Mentais na Infância, Dislexia e Psicodiagnóstico (Yazigi, 1996).

Em 1978 ingressou como chefe do Setor de Psicologia no Departamento de Psiquiatria e Psicologia Médica da então Escola Paulista de Medicina, hoje Unifesp, onde ministrou diversos cursos para as faculdades de medicina, de enfermagem, de fonoaudiologia e de tecnologia oftálmica, para médicos residentes e para e psicólogos em programa de especialização. No curso de pós-graduação de Psiquiatria e Psicologia Médica ministrou várias disciplinas e orientou 36 monografias de mestrado e 19 teses de doutorado e supervisionou sete estágios de pós-doutorado, além de inúmeras pesquisas de iniciação científica e de outra natureza. Entre 1986 até 1997 foi coordenadora do Curso de Especialização em Psicologia da Saúde, no qual também era professora de Psicodiagnóstico. Este curso foi criado graças ao empenho da professora, para o qual teve uma importante atuação para o status de importância que veio a representar. Também neste período em que lá esteve participou de comissões, assessoria à pró-reitoria, de cargos acadêmico-administrativos e da câmara técnica. Foi coordenadora da Comissão de Residência Multiprofissional em Atenção Básica e Saúde Mental. Destes cursos, e das teses orientadas, uma boa parte foi dedicada ao ensino e desenvolvimento de pesquisas na área de Avaliação Psi- cológica. Mas acima de tudo, a Profa. Latife Yazigi muito se dedicou e deu uma grande contribuição para a construção do papel e engrandeceu a imagem do psicólogo clínico em uma Instituição da Saúde, para a atuação do psicólogo na área da saúde mental, trazendo um novo olhar para o trabalho do psicólogo e como este pode contribuir para a saúde em geral. Foram muitos anos de 1978 e 2014 - de dedicação e contribuição para a Psicologia. Transformava os pedidos de trabalhos, de atendimentos, em teses e mostrava o papel sofisticado que um psicólogo pode desempenhar. Foi responsável pelo reconhecimento do psicólogo na instituição de saúde e dentro da psiquiatria. Entre as atividades desempenhadas pelos psicólogos nesta instituição, destacou-se a introdução da avaliação psicológica no contexto hospitalar e da psiquiatria.

Esta contribuição também inclui sua grande dedicação à pesquisa em Psicologia, Suas pesquisas têm um grande valor e contribuíram muito para a validação de instrumentos de avaliação psicológica, com predileção para o Rorschach e para o conhecimento de reações humanas. A seriedade de suas pesquisas tem representado o Brasil em diversas sociedades científicas internacionais. Tem sido uma grande pesquisadora da área. Destacam-se as inúmeras bolsas de pesquisas recebidas da Fundação de Amparo à Pesquisa do Estado de São Paulo (Fapesp) para si e para seus orientandos e as bolsas do Conselho Nacional de Desenvolvimento Científico e Tecnológico (CNPq), onde é pesquisadora $1 \mathrm{~B}$.

No início de sua carreira, seus trabalhos foram mais voltados ao conhecimento do funcionamento cerebral e sua relação com o funcionamento psicológico. Como exemplo de trabalho nesta área, temos "A Prova de Rorschach, a Epilepsia Temporal e a Especialização Hemisférica" (Yazigi, 1990). Por muitos anos também nesta área de conhecimento, desenvolveu a pesquisa "O Rorschach Bissimétrico e Assimetria Cerebral", como também estudou o "Teste das Faces Quiméricas”. Estas duas últimas pesquisas foram uma continuidade de sua formação em Chicago (Yazigi 1995).

Nos últimos anos (desde 2006) vem trabalhando com pesquisas sobre "Avaliação de Psicoterapia Psicanalítica por meio do Método de Rorschach e da Escala Wechsler de Inteligência em Serviço Público Universitário". Estas pesquisas iniciaram com o Sistema Compreensivo do Rorschach e a partir de 2010 passaram a ser realizadas por meio do R-PAS, cuja primeira publicação é de 2011. Este fato mos- 
tra como a Professora vem trabalhando em temas, procedimentos e instrumentos sempre muito atualizados. Para sua realização Profa. Latife recebeu financiamentos da Fapesp e do CNPq. Estes projetos de pesquisas vêm sendo sucessivamente renovados e os pesquisadores fazem avaliação psicológica das pessoas no início do processo psicoterapêutico e reavaliações anuais para o estudo, que são reavaliadas anualmente para o estudo das modificações no funcionamento mental obtidas pelo processo psicoterapêutico e a apreensão destes fenômenos na avaliação psicológica, estudando a eficácia da psicoterapia e quanto os testes psicológicos contribuem para este conhecimento. Seu último projeto nesta linha de pesquisa foi de 2014 a 2018, recém-terminado.

Profa. Latife sempre foi além do óbvio, com Altimari (Yazigi, \& Altimari, 1993) e com Nashat (Yazigi, \& Nashat, 2012), da Inglaterra escreveu sobre Bion pela ótica do Rorschach, integrando a Psicanálise com o instrumento de avaliação psicológica. Umas poucas palavras para uma outra faceta da Profa. Latife. Amante da Psicologia e das artes, em muitos momentos conseguiu construir este vínculo, como em seu interessante trabalho sobre Arthur Bispo do Rosário (Yazigi, 2012a,b), a apresentação de Modigliani et Botero, com Julieta Lagomarsino (Lagomarsino, \& Yazigi, 2002), ou o trabalho sobre Leonardo da Vinci e suas máquinas, em confronto com o método de Rorschach.

Desta forma, podemos dizer que a Profa. Latife Yazigi conquistou para si e para os que a acompanham, seja como alunos, orientandos, supervisionandos, leitores, colegas e amigos, um cabedal de experiência, oportunidades, conhecimentos e postura diante do conhecimento e da vida. Foi e continua sendo mentora de muitos psicólogos. Tem mostrado na vida uma busca incessante de conhecimentos. Podemos dizer que sua vida e seu trabalho têm dado uma enorme contribuição para os testes e para a área da Avaliação Psicológica.

\section{Agradecimento}

Agradeço à Profa. Dra, Norma Lottenberg Semer pela contribuição na redação deste texto, fornecendo material e informações sobre a Profa. Latife Yazigi.

\section{Referências}

Lagomarsino, J., \& Yazigi, L (2002) . Modigliani et Botero: corps à corps. Symposium International: L'empreite, la trace, la marque, Paris, França.

Yazigi, L. (1990) Changing process: case of a psychotic epileptic patient submitted to left temporal lobectomy. International Rorschach Congress, 1990, Paris, França, 13.

Yazigi, L. (2002a) L'imaginaire d'un malade psychotique et son expression dans ses travaux: Bispo do Rosario et le tour du monde du Manteau de la Présentation. Congrès de I'ARIC - Association pour la Recherche Interculturelle, Amiens, França, 9.

Yazigi, L. (2002b). Le monde magique d'Arthur Bispo do Rosario. Symposium International: L'empreite, la trace, la marque, Paris, França.

Yazigi, L. (1994). Memorial (Livre Docência). Escola Paulista de Medicina, São Paulo, SP, Brasil.

Yazigi, L. (1995). Rorschach and cerebral asymmetry. European Congress of Psychology, 1995, Atenas, Grécia, 4.

Yazigi, L., \& Altimari, M. B. (1993). Rorschach and Bion. International Rorschach Congress, 1993, Lisboa, Portugal, 14.

Yazigi, L., \& Nashat, S. (2012). Learning from the inkblot. Rorschachiana, 33(special issue), 214-235.

Yazigi, L., \& Succar, I. Z. (1987). Rorschachiana XVI. International Congress of Rorschach and other Projective Techniques., Guaruja, São Paulo, SP, Brazil, 12.

\section{Regina Sonia Gattas Fernandes do Nascimento}

Professora associada do Curso de Psicologia da Pontifícia Universidade Católica de São Paulo (PUC-SP), São Paulo - SP. Brasil. 
Psicologia: Ciência e Profissão 2018 v. 38 (núm. esp.), 182-188.

Endereço para envio de correspondência:

Rua Monte Alegre, 984.

CEP: 05014-901 Perdizes, São Paulo/SP

Recebido 25/07/2018

Aprovado 08/08/2018

Received $07 / 25 / 2018$

Approved 08/08/2018

Recibido 25/07/2018

Aceptado 08/08/2018

Como citar: Nascimento, R. S. G. F. (2018). Latife Yazigi: Abertura para o Mundo e para a Profissão.

Psicologia: Ciência e Profissão, 38(n.spe), 182-188. https://doi.org/10.1590/1982-3703000209316

How to cite: Nascimento, R. S. G. F. (2018). Latife Yazigi: an Opening to the World and to the Profession. Psicologia: Ciência e Profissão, 38(n.spe), 182-188. https://doi.org/10.1590/1982-3703000209316

Cómo citar: Nascimento, R. S. G. F. (2018). Latife Yazigi: apertura para el mundo y para la profesión. Psicologia: Ciência e Profissão, 38(n.spe), 182-188. https://doi.org/10.1590/1982-3703000209316 